\title{
The Association between Gut Microbiota and Intestinal Toll-like Receptors Genes Expression in Adenomatous and Colorectal Cancerous patients
}

\section{Sama Rezasoltani ( Samasoltani70@gmail.com )}

Department of Biology, Science and Research Branch, Islamic Azad University, Tehran, Iran https://orcid.org/00000003-0925-6486

\section{Maryam Sharafkhah}

Tehran University of Medical Sciences

\section{Hamid Asadzadeh Aghdaei}

Shaheed Beheshti University of Medical Sciences Research Institute for Gastroenterology and Liver Diseases

\section{Meysam Olfatifar}

Shaheed Beheshti University of Medical Sciences

\section{Ehsan Nazemalhosseini Mojarad}

Shaheed Beheshti University of Medical Sciences Research Institute for Gastroenterology and Liver Diseases

\section{Abbas Yadegar}

Shaheed Beheshti University of Medical Sciences Research Institute for Gastroenterology and Liver Diseases

\section{Mohammad Reza Zali}

Shaheed Beheshti University of Medical Sciences Research Institute for Gastroenterology and Liver Diseases

\section{Research}

Keywords: Adenomatous polyposis, colorectal cancer, gut microbiota, Toll-like receptors, intestinal inflammation

Posted Date: February 17th, 2020

DOI: https://doi.org/10.21203/rs.2.23741/v1

License: (c) (i) This work is licensed under a Creative Commons Attribution 4.0 International License. Read Full License 


\section{Abstract}

Background: Toll-like receptor (TLR) signaling has been implicated in colorectal cancer (CRC) development. Intestinal microbiota can affect the expression of TLRs, which may induce inflammatory responses and impair the gut homeostasis. Here, we aimed to evaluate certain intestinal microbiota related to TLRs expression in colonic tissues of adenomatous polyposis and CRC patients.

Results: Fecal and colonic tissue samples were collected from normal controls (NC), adenomatous (AP) cases and (CRC) patients via colonoscopy for CRC screening during 2016 to 2018 . Fecal samples were collected to analyze intestinal bacteria including Streptococcus bovis, Enterococcus faecalis , Bacteroides fragilis , enterotoxigenic Bacteroides fragilis (ETBF) , Fusobacterium nucleatum, Porphyromonas gingivalis, Porphyromonas spp . and Roseburia spp . by real-time PCR. Gene expression of TLR2, TLR4 and TLR5 was examined in colonic tissues by qRTPCR. Different abundant of gut bacteria were achieved in NC, AP and CRC groups. The genes expression of TLR2, TLR4 and TLR5 were significantly different in AP and CRC cases vs. normal group ( $P$ value $<0.05)$. There was a significant relationship between TLR2, TLR4, TLR5 genes expression and Roseburia spp., P. gingivalis and ETBF quantity in normal group. Also significant association between TLR2, TLR4 genes expression levels and the quantity of S.bovis , ETBF, Roseburia spp. and E. faecalis in AP and CRC cases were achieved.

Conclusion : Intestinal expression of TLR2, TLR4 and TLR5 is dynamic and depends on gut microbiota. Hence, altered immune activation in response to dysbiotic microbiota may promote intestinal inflammation in a group of patients with AP and CRC. Keyword: Adenomatous polyposis; colorectal cancer; gut microbiota; Toll-like receptors; intestinal inflammation

\section{Background:}

The relationship between cancer and epigenetic modification returns to four decades ago (1). With innovation new techniques to study epigenetic mechanism in gene expression modulation, epigenetics has become popular area of research in the field of cancer biology even cancer therapy (2). The most ubiquitous environmental factor in epigenetic modification is gut microbiota $(2,3)$. Gut microbiota has an important role in the biological microenvironment. Interestingly, $16 \%$ of cancers have been recognized to be caused by microbes and those associated to the liver and gastrointestinal (GI) tract are definitely identified as being microbe related $(3,4)$. Epigenetic regulation of oncogenes, proinflammatory mediators, tumor suppressor and miss match repair genes identify as significant mechanism by which homeostatic balance is lost and gut microbiota dysbiosis is occurred (2). Gut microbiota may play a critical role in progression of colorectal cancer (CRC) via their metabolite or their structural component which interacting with pathogen-associated molecular patterns (PAMP) and microbeassociated molecular pattern (MAMP) receptors such as Toll-like receptors (TLRs) (4). TLRs not only detect invading microbes but also recognize intracellular abnormality and mount an immune response, hence playing an important role in the human immune system homeostasis $(5,6)$. The abnormal activation of TLRs can compromise normal physiological processes and cause different types of disorders such as inflammatory diseases, autoimmune disorders and cancer $(7,8)$. Actually, there is a well-established link between TLR-induced inflammation and cancer development (9). Also, TLRs are recognized to play vital roles in CRC that affects the colon and the rectum (10). Colon and rectum is highly-populated by gut microbiota, emphasizing the critical role of TLRs in CRC pathogenesis (11). To maintain both locally and systemically gut homeostasis, tolerance must be achieved by the induction of anti-inflammatory molecules. Commensal bacteria are the vital regulators of the innate immune system, and can maintain homeostasis by stimulating antibody production and activating immune cells (12-14). 
Herein we tried to determine the relative expression level of TLR2, TLR5 and TLR4 (some critical TLRs during CRC initiation and progression) in adenomatous polyp and CRC cases compared to normal participants also checked the association between the expression of these important TLRs and certain gut bacteria which are identified in our previous studies $(15,16)$ during CRC initiation and progression.

\section{Results:}

According to the results were obtained for TLRs mRNA expression level, lower expression level of TLR5 (P-value = 0/033) and higher expression level of TLR2 and TLR4 (P-value = 0/000) in CRC cases compared to NC participants were achieved. Also AP cases and control groups were compared. Actually, decreased level of TLR5 (P-value = 0/041) mRNA expression and increased level of TLR2 and TLR4 mRNA (P-value = 0/003) were obtained in AP cases in contrast to NC subjects (Fig. 1). Moreover, based on our previous study and with evaluating bacterial quantification in stool samples of participants, increasing level of F. nucleatum $(P<0.001)$, E. faecalis $(P<0.001)$, Porphyromonas spp. $(P<0.001)$, P. gingivalis $(P<0.005)$ were achieved in CRC patients compared to AP cases, whereas $S$. bovis $(P<0.001)$, ETBF $(P<0.001)$, B. fragilis $(P<0.015)$ have shown statistically increased range in $C R C$ and AP compared to NC individuals (15). In the following, the relationship between demographic characterization of participants consisting sex, age, family history, GI history, smoking, food regime and physical activity and mRNA expression level of selected TLRs were investigated (Table 1). 
Table 1

The association between demographic characterization of individuals and TLRs mRNA expression level

\begin{tabular}{|c|c|c|c|}
\hline \multirow[t]{2}{*}{ Characteristics (Number) } & TLR2 RQ & TLR4 RQ & TLR5 RQ \\
\hline & Mean (SD) & Mean (SD) & Mean (SD) \\
\hline \multirow{3}{*}{$\begin{array}{l}\text { Sex } \\
\text { Female (41) } \\
\text { Male (52) }\end{array}$} & $5 / 8(4 / 1)$ & $7 / 1(4 / 7)$ & $0 / 70(0 / 58)$ \\
\hline & $6 / 5(4 / 07)$ & $7 / 7(4 / 5)$ & $0 / 64(0 / 62)$ \\
\hline & $P$ - value $=0 / 4$ & $P$ - value $=0 / 5$ & $P$ - value $=0 / 5$ \\
\hline \multirow{2}{*}{$\begin{array}{l}\text { Age } \\
>50 \\
<50\end{array}$} & $\begin{array}{l}5 / 8(4 / 3) \\
6 / 3(4 / 0)\end{array}$ & $\begin{array}{l}6 / 8(4 / 7) \\
7 / 7(4 / 6)\end{array}$ & $\begin{array}{l}0 / 75(0 / 62) \\
0 / 64(0 / 59)\end{array}$ \\
\hline & $P$ - value $=0 / 5$ & $P$ - value $=0 / 4$ & $P$ - value $=0 / 4$ \\
\hline \multirow{3}{*}{$\begin{array}{l}\text { Family history } \\
\text { No }(79) \\
\text { Yes (14) } \\
\text { Total (93) }\end{array}$} & $5 / 9(4 / 3)$ & $7 / 0(4 / 9)$ & $0 / 73(0 / 6)$ \\
\hline & $/ / 0(2 / 3)$ & $9 / 1(2 / 8)$ & $0 / 3 /(0 / 27)$ \\
\hline & $P$ - value $=0.63$ & $\mathrm{P}$ - value $=0.55$ & $P$ - value $=0.26$ \\
\hline \multirow{2}{*}{$\begin{array}{l}\text { GI history } \\
\text { No (24) } \\
\text { Yes }(69) \\
\text { Total (93) }\end{array}$} & $\begin{array}{l}5 / 6(4 / 2) \\
6 / 4(4 / 0)\end{array}$ & $\begin{array}{l}6 / 8(4 / 9) \\
7 / 7(4 / 5)\end{array}$ & $\begin{array}{l}0 / 83(0 / 67) \\
0 / 61(0 / 57)\end{array}$ \\
\hline & $P$ - value $=0.25$ & $P$ - value $=0.69$ & $P$ - value $=0.10$ \\
\hline \multirow{2}{*}{$\begin{array}{l}\text { Smoking } \\
\text { No (80) } \\
\text { Yes (13) } \\
\text { Total (93) }\end{array}$} & $\begin{array}{l}49 / 57 \\
31 / 19\end{array}$ & $\begin{array}{l}49 / 26 \\
33 / 12\end{array}$ & $\begin{array}{l}45 / 6 \\
55 / 2 \\
0.02\end{array}$ \\
\hline & $P$ - value $=0.02$ & $P$ - value $=0.045$ & $P$ - value $=0.045$ \\
\hline \multirow{2}{*}{$\begin{array}{l}\text { Food regime } \\
\text { All food ( } 79) \\
\text { Low consumption of vegetable and fruits (14) } \\
\text { Total (93) }\end{array}$} & $\begin{array}{l}8 / 8(3 / 3) \\
5 / 8(4 / 06)\end{array}$ & $\begin{array}{l}10 / 8(3 / 1) \\
6 / 9(4 / 6)\end{array}$ & $\begin{array}{l}0 / 30(0 / 27) \\
0 / 73(0 / 63)\end{array}$ \\
\hline & $P$ - value $=0.014$ & $P$ - value $=0.003$ & $P$ - value $=0.016$ \\
\hline Physical activity & $6 / 4(4 / 2)$ & $5 / 9(3 / 9)$ & $6 / 4(3 / 9)$ \\
\hline \multirow{2}{*}{$\begin{array}{l}\text { No }(64) \\
\text { Moderate (20) } \\
\text { Sever (9) } \\
\text { Total (93) }\end{array}$} & $\begin{array}{l}7 / 6(4 / 7) \\
0 / 6(0 / 59)\end{array}$ & $\begin{array}{l}7 / 2(4 / 6) \\
0 / 69(0 / 62)\end{array}$ & $\begin{array}{l}7 / 1(4 / 8) \\
0 / 6(0 / 66)\end{array}$ \\
\hline & $P$ - value $=0.54$ & $P$ - value $=0.83$ & $P$ - value $=0.70$ \\
\hline
\end{tabular}

Table 1: The association between demographic characterization of individuals and TLRs mRNA expression level 


\begin{tabular}{|c|c|c|c|}
\hline Characteristics (Number) & $\begin{array}{l}\text { TLR2 RQ } \\
\text { Mean (SD) }\end{array}$ & $\begin{array}{l}\text { TLR4 RQ } \\
\text { Mean (SD) }\end{array}$ & $\begin{array}{l}\text { TLR5 RQ } \\
\text { Mean (SD) }\end{array}$ \\
\hline \multirow{2}{*}{$\begin{array}{l}\text { Sex } \\
\text { Female (41) } \\
\text { Male (52) }\end{array}$} & $\begin{array}{l}5 / 8(4 / 1) \\
6 / 5(4 / 07)\end{array}$ & $\begin{array}{l}7 / 1(4 / 7) \\
7 / 7(4 / 5)\end{array}$ & $\begin{array}{l}0 / 70(0 / 58) \\
0 / 64(0 / 62)\end{array}$ \\
\hline & $P$-value $=0 / 4$ & $P$-value $=0 / 5$ & $P$ - value $=0 / 5$ \\
\hline \multirow[t]{3}{*}{$\begin{array}{l}\text { Age } \\
>50 \\
<50\end{array}$} & & & \\
\hline & $\begin{array}{l}5 / 8(4 / 3) \\
6 / 3(4 / 0)\end{array}$ & $\begin{array}{l}6 / 8(4 / 7) \\
7 / 7(4 / 6)\end{array}$ & $\begin{array}{l}0 / 75(0 / 62) \\
0 / 64(0 / 59)\end{array}$ \\
\hline & $P$ - value $=0 / 5$ & $P$-value $=0 / 4$ & $P$-value $=0 / 4$ \\
\hline \multirow{2}{*}{$\begin{array}{l}\text { Family history } \\
\text { No (79) } \\
\text { Yes (14) } \\
\text { Total (93) }\end{array}$} & $\begin{array}{l}5 / 9(4 / 3) \\
7 / 0(2 / 3)\end{array}$ & $\begin{array}{l}7 / 0(4 / 9) \\
9 / 1(2 / 8)\end{array}$ & $\begin{array}{l}0 / 73(0 / 6) \\
0 / 37(0 / 27)\end{array}$ \\
\hline & $P$-value $=0.63$ & $P$ - value $=0.55$ & $P$ - value $=0.26$ \\
\hline \multirow{2}{*}{$\begin{array}{l}\text { GI history } \\
\text { No (24) } \\
\text { Yes }(69) \\
\text { Total (93) }\end{array}$} & $\begin{array}{l}5 / 6(4 / 2) \\
6 / 4(4 / 0)\end{array}$ & $\begin{array}{l}6 / 8(4 / 9) \\
7 / 7(4 / 5)\end{array}$ & $\begin{array}{l}0 / 83(0 / 67) \\
0 / 61(0 / 57)\end{array}$ \\
\hline & $P$ - value $=0.25$ & $P$ - value $=0.69$ & $P$-value $=0.10$ \\
\hline \multirow[t]{2}{*}{$\begin{array}{l}\text { Smoking } \\
\text { No ( } 80) \\
\text { Yes }(13) \\
\text { Total (93) }\end{array}$} & $\begin{array}{l}49 / 57 \\
31 / 19\end{array}$ & $\begin{array}{l}49 / 26 \\
33 / 12\end{array}$ & $\begin{array}{l}45 / 6 \\
55 / 2 \\
0.02\end{array}$ \\
\hline & $P$ - value $=0.02$ & $P$-value $=0.045$ & $P$ - value $=0.045$ \\
\hline \multirow{2}{*}{$\begin{array}{l}\text { Food regime } \\
\text { All food (79) } \\
\text { Low consumption of vegetable and fruits (14) } \\
\text { Total (93) }\end{array}$} & $\begin{array}{l}8 / 8(3 / 3) \\
5 / 8(4 / 06)\end{array}$ & $\begin{array}{l}10 / 8(3 / 1) \\
6 / 9(4 / 6)\end{array}$ & $\begin{array}{l}0 / 30(0 / 27) \\
0 / 73(0 / 63)\end{array}$ \\
\hline & $P$ - value $=0.014$ & $P$-value $=0.003$ & P- value $=0.016$ \\
\hline \multirow{2}{*}{$\begin{array}{l}\text { Physical activity } \\
\text { No }(64) \\
\text { Moderate }(20) \\
\text { Sever }(9) \\
\text { Total (93) }\end{array}$} & $\begin{array}{l}6 / 4(4 / 2) \\
7 / 6(4 / 7) \\
0 / 6(0 / 59)\end{array}$ & $\begin{array}{l}5 / 9(3 / 9) \\
7 / 2(4 / 6) \\
0 / 69(0 / 62)\end{array}$ & $\begin{array}{l}6 / 4(3 / 9) \\
7 / 1(4 / 8) \\
0 / 6(0 / 66)\end{array}$ \\
\hline & $P$ - value $=0.54$ & $P$-value $=0.83$ & $P$ - value $=0.70$ \\
\hline \multicolumn{4}{|c|}{$\begin{array}{l}\text { Interestingly, there was significant relationship between food regimes and smoking habit of participants and } \\
\text { TLR2, TLR4 and TLR5 mRNA expression level in their colonic tissue. At last, the relationship between targeted } \\
\text { TLRs mRNA expression level and selected gut bacterial quantity based on CT was evaluated in different groups } \\
\text { of participants by pearson correlation analysis and computing P-value (Table 2). }\end{array}$} \\
\hline
\end{tabular}

Table 2: The relationship between TLR2, TLR4 and TLR5 mRNA expression level and selected gut bacterial quantity based on CT in normal, AP and CRC groups 


\begin{tabular}{|c|c|c|c|c|c|c|c|c|}
\hline $\begin{array}{l}\text { TLRs RQ } \\
\text { pearson } \\
\text { correlation } \\
\text { in groups }\end{array}$ & $\begin{array}{l}\text { F. } \\
\text { nucleatum } \\
\text { CT }\end{array}$ & $\begin{array}{l}\text { E. } \\
\text { faecalis } \\
\text { CT }\end{array}$ & $\begin{array}{l}\text { S. } \\
\text { bovis } \\
\text { CT }\end{array}$ & $\begin{array}{l}\text { P. } \\
\text { gingivalis } \\
\text { CT }\end{array}$ & $\begin{array}{l}\text { ETBF } \\
\text { CT }\end{array}$ & $\begin{array}{l}\text { B. } \\
\text { fragilis } \\
\text { CT }\end{array}$ & $\begin{array}{l}\text { Roseburia } \\
\text { Spp. } \\
\text { CT }\end{array}$ & $\begin{array}{l}\text { Porphyromonas } \\
\text { Spp. } \\
\text { CT }\end{array}$ \\
\hline $\begin{array}{l}\text { Normal } \\
\text { (31) }\end{array}$ & -0.092 & -0.07 & -0.12 & 0.5 & -0.022 & 0.17 & 0.4 & 0.32 \\
\hline TLR2 & $\begin{array}{l}\text { P-value }= \\
0.6\end{array}$ & $\begin{array}{l}\mathrm{P}- \\
\text { value= } \\
0.7\end{array}$ & $\begin{array}{l}\text { P- } \\
\text { value= } \\
0.5\end{array}$ & $\begin{array}{l}\text { P-value }= \\
0.003\end{array}$ & $\begin{array}{l}\text { P- } \\
\text { value= } \\
0.9\end{array}$ & $\begin{array}{l}\text { P- } \\
\text { value= } \\
0.3\end{array}$ & $\begin{array}{l}\text { P-value= } \\
0 / 03\end{array}$ & $P$ - value $=0.07$ \\
\hline $\begin{array}{l}\text { Normal } \\
\text { (31) }\end{array}$ & $-0 / 13$ & $-0 / 28$ & -0.09 & 0.52 & 0.1 & $-0 / 02$ & 0.53 & $0 / 11$ \\
\hline TLR4 RQ & $\begin{array}{l}\text { P-value= } \\
0.46\end{array}$ & $\begin{array}{l}\mathrm{P}- \\
\text { value= } \\
0.11\end{array}$ & $\begin{array}{l}P- \\
\text { value= } \\
0.62\end{array}$ & $\begin{array}{l}\text { P-value= } \\
0.003\end{array}$ & $\begin{array}{l}P- \\
\text { value= } \\
0.59\end{array}$ & $\begin{array}{l}P- \\
\text { value= } \\
0.99\end{array}$ & $\begin{array}{l}\text { P-value= } \\
0.002\end{array}$ & $\begin{array}{l}P-\text { value }= \\
0.55\end{array}$ \\
\hline $\begin{array}{l}\text { Normal } \\
\text { (31) }\end{array}$ & -0.18 & 0.176 & $0 / .74$ & 0.251 & 0.351 & $-0 / 063$ & -0.16 & $-0 / 097$ \\
\hline TLR5 & $\begin{array}{l}\text { P-value }= \\
0.31\end{array}$ & $\begin{array}{l}P- \\
\text { value= } \\
0.34\end{array}$ & $\begin{array}{l}\text { P- } \\
\text { value } \\
=0.69\end{array}$ & $\begin{array}{l}\text { P-value= } \\
0.17\end{array}$ & $\begin{array}{l}\text { P- } \\
\text { value= } \\
0.05\end{array}$ & $\begin{array}{l}\text { P- } \\
\text { value= } \\
0.7\end{array}$ & $\begin{array}{l}\text { P-value= } \\
0.37\end{array}$ & $\begin{array}{l}\text { P-value = } \\
0.6\end{array}$ \\
\hline AP (42) & 0.26 & 0.009 & 0.35 & -0.021 & 0.34 & -0.069 & 0.052 & $-/ 041$ \\
\hline TLR2 & $\begin{array}{l}\text { P-value= } \\
0.08\end{array}$ & $\begin{array}{l}\mathrm{P}- \\
\text { value= } \\
0.9\end{array}$ & $\begin{array}{l}P- \\
\text { value= } \\
0.02\end{array}$ & $\begin{array}{l}\text { P-value }= \\
0.8\end{array}$ & $\begin{array}{l}\text { P- } \\
\text { value= } \\
0.02\end{array}$ & $\begin{array}{l}\text { P- } \\
\text { value= } \\
0.6\end{array}$ & $\begin{array}{l}\mathrm{P} \text {-value= } \\
0 / 742\end{array}$ & $\begin{array}{l}P-\text { value }= \\
0.7\end{array}$ \\
\hline AP (42) & $0 / 16$ & 0.06 & 0.022 & -0.25 & 0.15 & 0.028 & -0.31 & 0.61 \\
\hline TLR4 & $\begin{array}{l}\text { P-value= } \\
0 / 31\end{array}$ & $\begin{array}{l}\mathrm{P}- \\
\text { value= } \\
0.7\end{array}$ & $\begin{array}{l}P- \\
\text { value= } \\
0.89\end{array}$ & $\begin{array}{l}\text { P-value= } \\
0.102\end{array}$ & $\begin{array}{l}P- \\
\text { value= } \\
0.33\end{array}$ & $\begin{array}{l}P- \\
\text { value= } \\
0.8\end{array}$ & $\begin{array}{l}\text { P-value= } \\
0.04\end{array}$ & $\begin{array}{l}\text { P-value= } \\
0.7\end{array}$ \\
\hline AP (42) & 0.061 & -0.11 & 0.23 & -0.001 & -0.021 & 0.18 & 0.19 & -0.13 \\
\hline TLR5 & $\begin{array}{l}\text { P-value }= \\
0.7\end{array}$ & $\begin{array}{l}\mathrm{P}- \\
\text { value= } \\
0.47\end{array}$ & $\begin{array}{l}P- \\
\text { value= } \\
0.13\end{array}$ & $\begin{array}{l}\text { P-value= } \\
0.997\end{array}$ & $\begin{array}{l}\text { P- } \\
\text { value= } \\
0.89\end{array}$ & $\begin{array}{l}\text { P- } \\
\text { value= } \\
0.24\end{array}$ & $\begin{array}{l}\text { P-value= } \\
0.22\end{array}$ & $\begin{array}{l}P-\text { value }= \\
0.4\end{array}$ \\
\hline CRC (20) & -0.07 & -0.23 & 0.4 & -0.013 & -0.014 & 0.06 & 0.19 & -0.40 \\
\hline TLR2 & $\begin{array}{l}\text { P- value }= \\
0.7\end{array}$ & $\begin{array}{l}P- \\
\text { value = } \\
0.32\end{array}$ & $\begin{array}{l}\text { P- } \\
\text { value } \\
=0.05\end{array}$ & $\begin{array}{l}\text { P-value } \\
=0.95\end{array}$ & $\begin{array}{l}\text { P- } \\
\text { value } \\
=0.95\end{array}$ & $\begin{array}{l}\text { P- } \\
\text { value= } \\
0.79\end{array}$ & $\begin{array}{l}\text { P- value= } \\
0.41\end{array}$ & $\begin{array}{l}\text { P-value= } \\
0.08\end{array}$ \\
\hline CRC (20) & -0.09 & 0.5 & 0.1 & -0.013 & 0.17 & 0.29 & 0.26 & 0.19 \\
\hline
\end{tabular}

Ithere is an inverse relationship between CT rate and bacterial quantity. The lower the CT, the greater the bacterial quantity.

Importantly there is an inverse relationship between CT rate and bacterial quantity. The lower the $\mathrm{CT}$, the greater the bacterial quantity. Surprisingly, there was a significant relationship between TLR2, TLR4 mRNA expression levels and the quantity of Roseburia spp., P. gingivalis $(P$-value $<0.05)$ and between TLR5mRNA expression levels and the quantity of ETBF in normal participants. Moreover, the significant association between TLR2 mRNA expression level and the quantity of S.bovis and ETBF, TLR4 mRNA expression level and Roseburia spp. quantity were obtained in AP cases. At last, in CRC cases, the significant relevance between TLR2 mRNA expression and S.bovis quantity, TLR4 mRNA expression and E. faecalis quantity were achieved. 


\begin{tabular}{|c|c|c|c|c|c|c|c|c|}
\hline $\begin{array}{l}\text { TLRs RQ } \\
\text { pearson } \\
\text { correlation } \\
\text { in groups }\end{array}$ & $\begin{array}{l}\text { F. } \\
\text { nucleatum } \\
\text { CT }\end{array}$ & $\begin{array}{l}\text { E. } \\
\text { faecalis } \\
\text { CT }\end{array}$ & $\begin{array}{l}\text { S. } \\
\text { bovis } \\
\text { CT }\end{array}$ & $\begin{array}{l}\text { P. } \\
\text { gingivalis } \\
\text { CT }\end{array}$ & $\begin{array}{l}\text { ETBF } \\
\text { CT }\end{array}$ & $\begin{array}{l}\text { B. } \\
\text { fragilis } \\
\text { CT }\end{array}$ & $\begin{array}{l}\text { Roseburia } \\
\text { Spp. } \\
\text { CT }\end{array}$ & $\begin{array}{l}\text { Porphyromonas } \\
\text { Spp. } \\
\text { CT }\end{array}$ \\
\hline & $\begin{array}{l}\text { P-value }= \\
0.6\end{array}$ & $\begin{array}{l}P- \\
\text { value }= \\
0.009\end{array}$ & $\begin{array}{l}\text { P- } \\
\text { value } \\
=0.6\end{array}$ & $\begin{array}{l}\text { P-value } \\
=0.95\end{array}$ & $\begin{array}{l}\text { P- } \\
\text { value } \\
=0.45\end{array}$ & $\begin{array}{l}\text { P- } \\
\text { value } \\
=0.21\end{array}$ & $\begin{array}{l}\text { P- value }= \\
0.27\end{array}$ & $\begin{array}{l}\text { P-value= } \\
0.42\end{array}$ \\
\hline CRC (20) & -0.14 & 0.033 & -0.36 & -0.014 & -0.21 & -0.14 & -0.28 & 0.094 \\
\hline TLR5 & $\begin{array}{l}\mathrm{P} \text {-value }= \\
0.5\end{array}$ & $\begin{array}{l}P- \\
\text { value = } \\
0.88\end{array}$ & $\begin{array}{l}P \text { - } \\
\text { value } \\
=0.11\end{array}$ & $\begin{array}{l}P \text {-value } \\
=0.95\end{array}$ & $\begin{array}{l}P- \\
\text { value } \\
=0.35\end{array}$ & $\begin{array}{l}\text { P- } \\
\text { value= } \\
0.5\end{array}$ & $\begin{array}{l}P-\text { value }= \\
0.23\end{array}$ & $P$ - value $=0.6$ \\
\hline \multicolumn{9}{|c|}{$\begin{array}{l}\text { *There is an inverse relationship between } \mathrm{CT} \text { rate and bacterial quantity. The lower the } \mathrm{CT} \text {, the greater the } \\
\text { bacterial quantity. }\end{array}$} \\
\hline \multicolumn{9}{|c|}{$\begin{array}{l}\text { Importantly there is an inverse relationship between CT rate and bacterial quantity. The lower the CT, the greater } \\
\text { the bacterial quantity. Surprisingly, there was a significant relationship between TLR2, TLR4 mRNA expression } \\
\text { levels and the quantity of Roseburia spp., P. gingivalis (P- value }<0.05 \text { ) and between TLR5mRNA expression } \\
\text { levels and the quantity of ETBF in normal participants. Moreover, the significant association between TLR2 } \\
\text { mRNA expression level and the quantity of S.bovis and ETBF, TLR4 mRNA expression level and Roseburia spp. } \\
\text { quantity were obtained in AP cases. At last, in CRC cases, the significant relevance between TLR2 mRNA } \\
\text { expression and S.bovis quantity, TLR4 mRNA expression and E. faecalis quantity were achieved. }\end{array}$} \\
\hline
\end{tabular}


Table 2

The relationship between TLR2, TLR4 and TLR5 mRNA expression level and selected gut bacterial quantity based on CT in normal, AP and CRC groups

\begin{tabular}{|c|c|c|c|c|c|c|c|c|}
\hline $\begin{array}{l}\text { TLRs RQ } \\
\text { pearson } \\
\text { correlation } \\
\text { in groups }\end{array}$ & $\begin{array}{l}\text { F. } \\
\text { nucleatum } \\
\text { CT }\end{array}$ & $\begin{array}{l}\text { E. } \\
\text { faecalis } \\
\text { CT }\end{array}$ & $\begin{array}{l}\text { S. } \\
\text { bovis } \\
\text { CT }\end{array}$ & $\begin{array}{l}\text { P. } \\
\text { gingivalis } \\
\text { CT }\end{array}$ & $\begin{array}{l}\text { ETBF } \\
\text { CT }\end{array}$ & $\begin{array}{l}\text { B. } \\
\text { fragilis } \\
\text { CT }\end{array}$ & $\begin{array}{l}\text { Roseburia } \\
\text { Spp. } \\
\text { CT }\end{array}$ & $\begin{array}{l}\text { Porphyromonas } \\
\text { Spp. } \\
\text { CT }\end{array}$ \\
\hline $\begin{array}{l}\text { Normal } \\
\text { (31) }\end{array}$ & -0.092 & -0.07 & -0.12 & 0.5 & -0.022 & 0.17 & 0.4 & 0.32 \\
\hline TLR2 & $\begin{array}{l}\text { P-value }= \\
0.6\end{array}$ & $\begin{array}{l}\mathrm{P}- \\
\text { value= } \\
0.7\end{array}$ & $\begin{array}{l}\text { P- } \\
\text { value= } \\
0.5\end{array}$ & $\begin{array}{l}\text { P-value }= \\
0.003\end{array}$ & $\begin{array}{l}\mathrm{P}- \\
\text { value= } \\
0.9\end{array}$ & $\begin{array}{l}\mathrm{P}- \\
\text { value= } \\
0.3\end{array}$ & $\begin{array}{l}P-\text { value }= \\
0 / 03\end{array}$ & $P$ - value $=0.07$ \\
\hline $\begin{array}{l}\text { Normal } \\
(31)\end{array}$ & $-0 / 13$ & $-0 / 28$ & -0.09 & 0.52 & 0.1 & $-0 / 02$ & 0.53 & $0 / 11$ \\
\hline TLR4 RQ & $\begin{array}{l}\text { P-value= } \\
0.46\end{array}$ & $\begin{array}{l}P- \\
\text { value= } \\
0.11\end{array}$ & $\begin{array}{l}\mathrm{P}- \\
\text { value= } \\
0.62\end{array}$ & $\begin{array}{l}\text { P-value= } \\
0.003\end{array}$ & $\begin{array}{l}P- \\
\text { value= } \\
0.59\end{array}$ & $\begin{array}{l}\mathrm{P}- \\
\text { value= } \\
0.99\end{array}$ & $\begin{array}{l}\text { P-value= } \\
0.002\end{array}$ & $\begin{array}{l}\text { P-value= } \\
0.55\end{array}$ \\
\hline $\begin{array}{l}\text { Normal } \\
(31)\end{array}$ & -0.18 & 0.176 & $0 / .74$ & 0.251 & 0.351 & $-0 / 063$ & -0.16 & $-0 / 097$ \\
\hline TLR5 & $\begin{array}{l}\text { P-value }= \\
0.31\end{array}$ & $\begin{array}{l}\text { P- } \\
\text { value= } \\
0.34\end{array}$ & $\begin{array}{l}\text { P- } \\
\text { value } \\
=0.69\end{array}$ & $\begin{array}{l}\text { P-value }= \\
0.17\end{array}$ & $\begin{array}{l}\text { P- } \\
\text { value= } \\
0.05\end{array}$ & $\begin{array}{l}\text { P- } \\
\text { value= } \\
0.7\end{array}$ & $\begin{array}{l}\text { P-value }= \\
0.37\end{array}$ & $\begin{array}{l}\text { P-value = } \\
0.6\end{array}$ \\
\hline $\mathrm{AP}(42)$ & 0.26 & 0.009 & 0.35 & -0.021 & 0.34 & -0.069 & 0.052 & $-/ 041$ \\
\hline TLR2 & $\begin{array}{l}\text { P-value }= \\
0.08\end{array}$ & $\begin{array}{l}P- \\
\text { value= } \\
0.9\end{array}$ & $\begin{array}{l}P- \\
\text { value= } \\
0.02\end{array}$ & $\begin{array}{l}P-\text { value }= \\
0.8\end{array}$ & $\begin{array}{l}P- \\
\text { value= } \\
0.02\end{array}$ & $\begin{array}{l}\text { P- } \\
\text { value = } \\
0.6\end{array}$ & $\begin{array}{l}P-\text { value }= \\
0 / 742\end{array}$ & $\begin{array}{l}\text { P-value }= \\
0.7\end{array}$ \\
\hline AP (42) & $0 / 16$ & 0.06 & 0.022 & -0.25 & 0.15 & 0.028 & -0.31 & 0.61 \\
\hline TLR4 & $\begin{array}{l}P-\text { value }= \\
0 / 31\end{array}$ & $\begin{array}{l}\text { P- } \\
\text { value= } \\
0.7\end{array}$ & $\begin{array}{l}\mathrm{P}- \\
\text { value= } \\
0.89\end{array}$ & $\begin{array}{l}\text { P-value= } \\
0.102\end{array}$ & $\begin{array}{l}\mathrm{P}- \\
\text { value= } \\
0.33\end{array}$ & $\begin{array}{l}\mathrm{P}- \\
\text { value= } \\
0.8\end{array}$ & $\begin{array}{l}\text { P-value }= \\
0.04\end{array}$ & $\begin{array}{l}\text { P-value }= \\
0.7\end{array}$ \\
\hline $\mathrm{AP}(42)$ & 0.061 & -0.11 & 0.23 & -0.001 & -0.021 & 0.18 & 0.19 & -0.13 \\
\hline TLR5 & $\begin{array}{l}\text { P- value = } \\
0.7\end{array}$ & $\begin{array}{l}\text { P- } \\
\text { value= } \\
0.47\end{array}$ & $\begin{array}{l}P- \\
\text { value= } \\
0.13\end{array}$ & $\begin{array}{l}\text { P-value= } \\
0.997\end{array}$ & $\begin{array}{l}\text { P- } \\
\text { value= } \\
0.89\end{array}$ & $\begin{array}{l}\text { P- } \\
\text { value= } \\
0.24\end{array}$ & $\begin{array}{l}\text { P- value= } \\
0.22\end{array}$ & $\begin{array}{l}\text { P- value }= \\
0.4\end{array}$ \\
\hline CRC (20) & -0.07 & -0.23 & 0.4 & -0.013 & -0.014 & 0.06 & 0.19 & -0.40 \\
\hline TLR2 & $\begin{array}{l}P-\text { value = } \\
0.7\end{array}$ & $\begin{array}{l}\text { P- } \\
\text { value = } \\
0.32\end{array}$ & $\begin{array}{l}\text { P- } \\
\text { value } \\
=0.05\end{array}$ & $\begin{array}{l}\text { P-value } \\
=0.95\end{array}$ & $\begin{array}{l}\text { P- } \\
\text { value } \\
=0.95\end{array}$ & $\begin{array}{l}\text { P- } \\
\text { value= } \\
0.79\end{array}$ & $\begin{array}{l}\text { P- value= } \\
0.41\end{array}$ & $\begin{array}{l}\text { P-value= } \\
0.08\end{array}$ \\
\hline CRC (20) & -0.09 & 0.5 & 0.1 & -0.013 & 0.17 & 0.29 & 0.26 & 0.19 \\
\hline TLR4 & $\begin{array}{l}P \text { - value }= \\
0.6\end{array}$ & $\begin{array}{l}P- \\
\text { value = } \\
0.009\end{array}$ & $\begin{array}{l}P- \\
\text { value } \\
=0.6\end{array}$ & $\begin{array}{l}P \text {-value } \\
=0.95\end{array}$ & $\begin{array}{l}P \text { - } \\
\text { value } \\
=0.45\end{array}$ & $\begin{array}{l}\text { P- } \\
\text { value } \\
=0.21\end{array}$ & $\begin{array}{l}P-\text { value }= \\
0.27\end{array}$ & $\begin{array}{l}\text { P- value= } \\
0.42\end{array}$ \\
\hline
\end{tabular}

*There is an inverse relationship between $\mathrm{CT}$ rate and bacterial quantity. The lower the $\mathrm{CT}$, the greater the bacterial quantity. 


\begin{tabular}{|c|c|c|c|c|c|c|c|c|}
\hline $\begin{array}{l}\text { TLRs RQ } \\
\text { pearson } \\
\text { correlation } \\
\text { in groups }\end{array}$ & $\begin{array}{l}\text { F. } \\
\text { nucleatum } \\
\text { CT }\end{array}$ & $\begin{array}{l}\text { E. } \\
\text { faecalis } \\
\text { CT }\end{array}$ & $\begin{array}{l}\text { S. } \\
\text { bovis } \\
\text { CT }\end{array}$ & $\begin{array}{l}\text { P. } \\
\text { gingivalis } \\
\text { CT }\end{array}$ & $\begin{array}{l}\text { ETBF } \\
\text { CT }\end{array}$ & $\begin{array}{l}\text { B. } \\
\text { fragilis } \\
\text { CT }\end{array}$ & $\begin{array}{l}\text { Roseburia } \\
\text { Spp. } \\
\text { CT }\end{array}$ & $\begin{array}{l}\text { Porphyromonas } \\
\text { Spp. } \\
\text { CT }\end{array}$ \\
\hline CRC (20) & -0.14 & 0.033 & -0.36 & -0.014 & -0.21 & -0.14 & -0.28 & 0.094 \\
\hline TLR5 & $\begin{array}{l}\text { P-value }= \\
0.5\end{array}$ & $\begin{array}{l}P- \\
\text { value = } \\
0.88\end{array}$ & $\begin{array}{l}\text { P- } \\
\text { value } \\
=0.11\end{array}$ & $\begin{array}{l}\text { P-value } \\
=0.95\end{array}$ & $\begin{array}{l}P \text { - } \\
\text { value } \\
=0.35\end{array}$ & $\begin{array}{l}P- \\
\text { value= } \\
0.5\end{array}$ & $\begin{array}{l}P-\text { value }= \\
0.23\end{array}$ & $P$ - value $=0.6$ \\
\hline
\end{tabular}

*There is an inverse relationship between CT rate and bacterial quantity. The lower the CT, the greater the bacterial quantity.

\section{Discussion:}

In current study, it was focused on evaluating the expression level of the most critical TLRs genes including TLR2, TLR4 and TLR5 in AP and CRC cases compared to NC participants. Based on present study the expression levels of TLR2 and TLR4, were significantly increased in both AP and CRC cases which is particularly higher expression rate was achieved in $C R C$ individuals ( $P$ value $<0.0001$ ). According to relative expression level of these genes in both cases of AP and CRC, it was assumed that up regulation of TLR2 and TLR4 may be occurred in the first step of changing intestinal tissue in CRC initiation. Hence TLR2 and TLR4 may be offered as biomarkers for characterization the AP and CRC in the first step of CRC initiation (21). This result confirmed that TLR2 and TLR4, which have been shown to up regulate in the process of polyposis and carcinogenesis, may initially induced inflammation and increased immune system stimulation to the gram-negative bacterial structures, bacterial components and metabolites in the direction of more facilitated CRC initiation $(21,2)$. On the other hand, we have observed TLR5 mRNA expression level was decreased in AP and CRC participants compared to NC group. This means that TLR5 mRNA expression level may be essential for maintaining balance and gastrointestinal haemostasis and any imbalance or its reduction could lead the colon to inflammation and disease (22). Our results confirmed the Yang et al. study, which they declared in the healthy gut; TLR3 and TLR5 seem to be constitutively expressed, in contrast, TLR2 and TLR4 are expressed at very low levels, this claim suggesting that the expression of TLR3 and TLR5 receptors is mediated to avoid autoinflammatory immune activation in response to commensal bacteria (2). Also our results were in agree with Kelly et al. that demonstrated in the healthy gut, TLR3 and TLR5 appear to be constitutively expressed, while TLR2 and TLR4 are expressed at very low-level, actually, they inferred that the expression of TLR2 and TLR4 is carefully regulated to avoid unnecessary immune activation in response to the commensal bacteria (23). It is assumed that differences in immune outcome in response to pathogenic and commensal bacteria is associated to the MAMP structures found on commensals and pathogens are not functionally equivalent in regards to their ability to engage TLR signaling and activation. For example, some molecules of LPS are potent inducers of TLR4 signaling, while others are antagonistic $(23,24)$, or the ability of specific bacterial flagellins to activate the TLR5, while other structures are nonactivating (23). Above all Anton G. Kutikhin et al. also presented exactly the same results as ours regarding increased a mounts of TLR2 and TLR4 and decreased TLR5 mRNA expression in CRC and AP cases (22). In addition, there was a significant relationship between participant's food regimes, smoking habit and intestinal TLR2, TLR4 and TLR5 mRNA expression levels. This means that, the consumption of vegetables, fruits, meats, processed food and being smoker or not has a significant effect on the expression level of intestinal TLRs and maintenance of the haemostatic balance. One of the strength points of the current study is to investigate the relationship between gut microbiota and TLRs mRNA 
expression levels, that significant association between these TLRs mRNA expression levels and the abundance of gut bacteria such as P. gingivalis, Roseburia spp., S. bovis, ETBF and E. faecalis were achieved. These results were strongly in consistent with previous studies that proved the interaction between gut microbiota and TLRs affects homeostasis and immune responses or alteration of the gut microbiota is associated with immunological regulation and inflammation $(15,25,26)$. Above all, TLRs signaling has been implicated in the inflammatory responses in intestinal epithelial cells (IECs). These inflammatory signals regulate complicate interactions between TLRs and commensal microbes and are required for IEC proliferation, immune response and homeostasis. The upregulation of certain TLRs in CRC tissues suggests that TLRs may play an essential role in the prognosis of chronic and inflammatory diseases that develop CRC.

\section{Conclusions:}

Taking together, the current study depicted the up regulation of TLR2, TLR4 and down regulation of TLR5 in both AP and CRC cases and assumed that TLRs expression changes may be occurred during CRC initiation. In other words, TLRs signaling has been implicated in the inflammatory responses in IECs. These inflammatory responses mediate interactions between commensal microbiota and TLRs and are critically required for immune response and homeostasis. The upregulation and down regulation of certain TLRs in CRC tissues suggest that TLRs may have an essential role in the prognosis of chronic and inflammatory diseases that finally result CRC. In addition this study demonstrated that the intestinal expression of TLR2, TLR4 and TLR5 is dynamic and depends on the presence of commensal intestinal microbiota. Hence altered immune activation in response to dysbiotic microbiota may promote intestinal inflammation in patients with AP and CRC.

\section{Material And Methods:}

\section{Data collection for study population:}

This case-control study was conducted in 2016- 2018 on 93 eligible cases consisting CRC, AP and NC in Taleghani hospital, Tehran-Iran. Population only included participants without previous colon or rectal surgery, CRC, inflammatory or infectious injuries of the intestine. The study was approved by the Clinical Research Ethics Committee of the Shahid Beheshti University of Medical Sciences and the Ethics Committee of Taleghani Hospital, Tehran, Iran.

\section{Tissue sample collection:}

Colonic tissue samples were collected from participants at the time of colonoscopy and during the procedure. Written consent procedure and the study protocol were approved by the ethics committee of the Shahid Beheshti University of Medical Sciences, and the Ethics Committee of Taleghani Hospital, Tehran, Iran.

\section{Fecal sample collection:}

Fresh stool samples were collected two weeks to three days before colonoscopy and in all patients prior to bowel clean. Whole fresh stool samples were provided in sterile boxes, and $10 \mathrm{~g}$ was frozen at $-20 \mathrm{C} \Downarrow$. All samples were then selected for DNA extraction and absolute quantitative RT PCR.

\section{RNA extraction from tissue samples:}


RNA was extracted from the tissue surfaces by using TRIzo/ reagent. After final precipitation, the DNA was resuspended in TE buffer and stored at $-20 \mathrm{CD}$ for further analysis.

\section{DNA extraction from stool}

Genomic DNA was extracted from frozen preserved fecal samples using QIAamp DNA Stool Mini Kit (Qiagene, Hilden, Germany).

\section{Evaluating TLR2, TLR4 and TLR 5 mRNA Expression Level in Tissue Samples:}

RNAs were converted to cDNA by Retrotransciptase (RT) reaction Primescript TM RT Reagent kit (Takara- Japan). Following cDNA synthesis, to evaluate TLR2, TLR4 and TLR5 genes expression level, RT PCR and relative quantification method were performed by using Premix Ex Taq SYBR (Takara Bio- Japan) and Rotor Gene (QIAGENGermany). Real-time PCR was carried out by expressive primers $(17,18)$ under the following conditions: $95^{\circ} \mathrm{C}$ for $5 \mathrm{~s}$, 40 cycles of $95^{\circ} \mathrm{C}$ for $5 \mathrm{~s}, 60^{\circ} \mathrm{C}$ for $34 \mathrm{~s}, 95^{\circ} \mathrm{C}$ for $15 \mathrm{~s}, 60^{\circ} \mathrm{C}$ for $1 \mathrm{~s}$ and $60^{\circ} \mathrm{C}$ for $15 \mathrm{~s}$ [14]. Amplification signals for samples were normalized by $\beta-2$-microglobulin. Fold change of TLRs genes expression was evaluated by $2^{-\triangle \Delta C T}$ method (19).

\section{Evaluating Bacterial Quantification in Fecal Samples:}

Eight bacterial candidates which investigated based on our previous studies were quantified in stool samples of participants by absolute qRT -PCR (Syber Green detection system), including Streptococcus bovis/gallolyticus, Enterococcus faecalis, Bacteroides fragilis, Enterotoxigenic Bacteroides fragilis (ETBF), Fusobacterium nucleatum, Porphyromonas gingivalis, Porphyromonas spp. and Roseburia spp. DNA amplification was performed with ABI 7500 (Applied Biosystem) $(15,16,20)$.

\section{Statistical analysis:}

Descriptive statistics to describe the expression level of TLR2, TLR4 and TLR5 in terms of demographic characteristic variables were used. The Pearson correlation test was applied to measure the association between the gut bacterial quantity and TLRs expressions. We also used the t-student and one-way ANOVA tests or their nonparametric equivalents (mann-whitney and kruskal-wallis tests) to compare the gene expressions in terms of baseline variables. Subsequently, we applied the post hoc test for significant relationship.

\section{Abbreviations}

TLR

Toll-like receptor; CRC:colorectal cancer; NC:normal controls; AP:adenomatous polyposis; qPCR:real time quantitative PCR; ETBF:enterotoxigenic Bacteroides fragilis

\section{Declarations}

\section{Ethics approval and consent to participate}

In this study samples were collected following to the approval by Ethics Committee of Shahid Beheshti University of Medical Sciences. The informed consent was obtained in all cases. Ethics Approval Code: 
IR.SBMU.RIGLD.REC.1395.851

\section{Consent for publication:}

All authors have consented to publish the results described in this research article.

\section{Availability of data and material}

The data supporting the conclusions of this article are included within the article.

\section{Competing interests}

The authors declare that they have no competing interests.

\section{Funding sources}

This work was supported by Shahid Beheshti University of Medical Sciences (Project Number: RIGLD 851).

\section{Authors' contributions}

SR is first author who carried out all laboratory experiment, collected data and drafted the manuscript. MS and MO provided statistical analysis of the study. HA and MZ are the gastroenterologists who did colonoscopy and provided the specimens from all cases. ENM participated in the design of the study. AY revised the manuscript. All authors read and approved the final manuscript.

\section{Acknowledgements}

We would like to thank the vice chancellor of Shahid Beheshti University of Medical Sciences for supporting Project No. RIGLD 851.

\section{Authors' information}

- Gastroenterology and Liver Diseases Research Center, Research Institute for Gastroenterology and Liver Diseases, Shahid Beheshti University of Medical Sciences, Tehran, Iran. ${ }^{2-}$ Liver and Pancreatobiliary Diseases Research Center, Digestive Diseases Research Institute, Tehran University of Medical Sciences, Tehran, Iran. ${ }^{3-}$ Basic and Molecular Epidemiology of Gastrointestinal Disorders Research Center, Research Institute for Gastroenterology and Liver Diseases, Shahid Beheshti University of Medical Sciences, Tehran, Iran. ${ }^{4-}$ Foodborne and Waterborne Diseases Research Center, Research Institute for Gastroenterology and Liver Diseases, Shahid Beheshti University of Medical Sciences, Tehran, Iran

\section{References}

1. -Sharma S, Kelly TK, Jones PA. Epigenetics in cancer. Carcinogenesis. 2010;31:27-36.

2. -Yang T, Owen JL, Lightfoot YL, Kladde MP, Mohamadzadeh M. Microbiota impact on the epigenetic regulation of colorectal cancer. Trends Mol Med. 2013;19:714-25.

3. 
-Rezasoltani S, Asadzadeh-Aghdaei H, Nazemalhosseini-Mojarad E, Dabiri H, Ghanbari R, Zali MR. Gut microbiota, epigenetic modification and colorectal cancer. Iran J Microbiol. 2017;9:55-63.

4.

-Zeller G, Tap J, Voigt AY, Sunagawa S, Kultima JR, Costea PI, et al. Potential of fecal microbiota for early-stage detection of colorectal cancer. Mol Syst Biol. 2014;10:766.

5 .

-Yesudhas D, Gosu V, Anwar MA, Choi S. Multiple roles of toll-like receptor 4 in colorectal cancer. Front Immunol. 2014;15:334.

6.

-Spiljar M, Merkler D, Trajkovski M. The immune system bridges the gut microbiota with systemic energy homeostasis: focus on TLRs, mucosal barrier, and SCFAs. Front Immunol. 2017;8:1353.

7.

-Lavelle EC, Murphy C, O'Neill LA, Creagh EM. The role of TLRs, NLRs, and RLRs in mucosal innate immunity and homeostasis. Mucosal Immunol. 2010;3:17-28.

8.

-Navegantes KC, de Souza Gomes R, Pereira PAT, Czaikoski PG, Azevedo CHM, Monteiro MC. Immune modulation of some autoimmune diseases: the critical role of macrophages and neutrophils in the innate and adaptive immunity. $J$ Transl Med. 2017;15:36.

9.

-So EY, Ouchi T. The application of toll like receptors for cancer therapy. Int J Biol Sci. 2010;6:675-81.

10.

-Messaritakis I, Stogiannitsi M, Koulouridi A, Sfakianaki M, Voutsina A, Sotiriou A, et al. Evaluation of the detection of Toll-like receptors (TLRs) in cancer development and progression in patients with colorectal cancer. PLoS One. 2018;13:e0197327.

11.

-Paarnio K, Väyrynen S, Klintrup K, Ohtonen P, Mäkinen MJ, Mäkelä J, et al. Divergent expression of bacterial wall sensing Toll-like receptors 2 and 4 in colorectal cancer. World J Gastroenterol. 2017;23:4831-8.

12.

-Belkaid Y, Hand T. Microbiome, inflammation and cancer. Cancer J. 2014;20:181-9.

13.

-Yiu JH, Dorweiler B, Woo CW. Interaction between gut microbiota and toll-like receptor: from immunity to metabolism. J Mol Med (Berl). 2017;95:13-20.

14.

-Sun J, Kato I. Gut microbiota, inflammation and colorectal cancer. Genes Dis. 2016;3:130-43.

15.

-Rezasoltani S, Sharafkhah M, Asadzadeh Aghdaei H, Nazemalhosseini Mojarad E, Dabiri H, Akhavan Sepahi A, et al. Applying simple linear combination, multiple logistic and factor analysis methods for candidate fecal bacteria as novel biomarkers for early detection of adenomatous polyps and colon cancer. J Microbiol Methods. 2018;12:82-8. 16.

-Rezasoltani S, Asadzadeh Aghdaei H, Dabiri H, Akhavan Sepahi A, Modarressi MH, Nazemalhosseini Mojarad E. The association between fecal microbiota and different types of colorectal polyp as precursors of colorectal cancer. Microb Pathog. 2018;22:244-9.

17. 
-Kim TW, Lee SJ, Oh BM, Lee H, Uhm TG, Min JK, et al. Epigenetic modification of TLR4 promotes activation of NFKB by regulating methyl-CpG-binding domain protein 2 and Sp1 in gastric cancer. Oncotarget. 2016;7:4195-209. 18.

-Li H, Yang T, Li FY, Ning Q, Sun ZM. TLR4 overexpression inhibits endothelial PAS domain-containing protein 1 expression in the lower respiratory tract of patients with chronic COPD. Cell Physiol Biochem. 2016;39:685-92. 19.

-Peyravian N, Gharib E, Moradi A, Mobahat M, Tarban P, Azimzadeh P, et al. Evaluating the expression level of costimulatory molecules CD 80 and CD 86 in different types of colon polyps. Curr Res Transl Med. 2018;66:19-25. 20.

-Rezasoltani S, Dabiri H, Asadzadeh Aghdaei H, Sepahi AA, Modarressi MH, Mojarad EN. An improved real- time qPCR technique for quantification of intestinal bacteria in human fecal samples. South Asian J Exp Biol. 2017;7:2019.

21.

-Sekirov I, Russell SL, Antunes LC. Finlay BB. Gut microbiota in health and disease. Physiol Rev. 2010;90:859-904. 22.

Kutikhin -AG, Yuzhalin AE. Pattern recognition receptors and cancer. Front Immunol. 2015;6:481.

23.

-Kelly D, Mulder IE. Microbiome and immunological interactions. Nutr Rev. 2012;70:18-30.

24.

-Mogensen TH. Pathogen recognition and inflammatory signaling in innate immune defenses. Clin Microbiol Rev. $2009 ; 22: 240-73$.

25.

-Shukla R, Ghoshal U, Ranjan P, Ghoshal UC. Expression of toll-like receptors, Pro-, and anti-inflammatory cytokines in relation to gut microbiota in irritable bowel syndrome: The evidence for its micro-organic basis. J Neurogastroenterol Motil. 2018;24:628-42.

26.

-Inoue R, Yajima T, Tsukahara T. Expression of TLR2 and TLR4 in murine small intestine during postnatal development. Biosci Biotechnol Biochem. 2017;81:350-8.

Legends

Legends. of Fig. 1: TLR2, TLR4 and TLR5 mRNA expression levels in AP and CRC cases compared to normal participants.

\section{Figures}




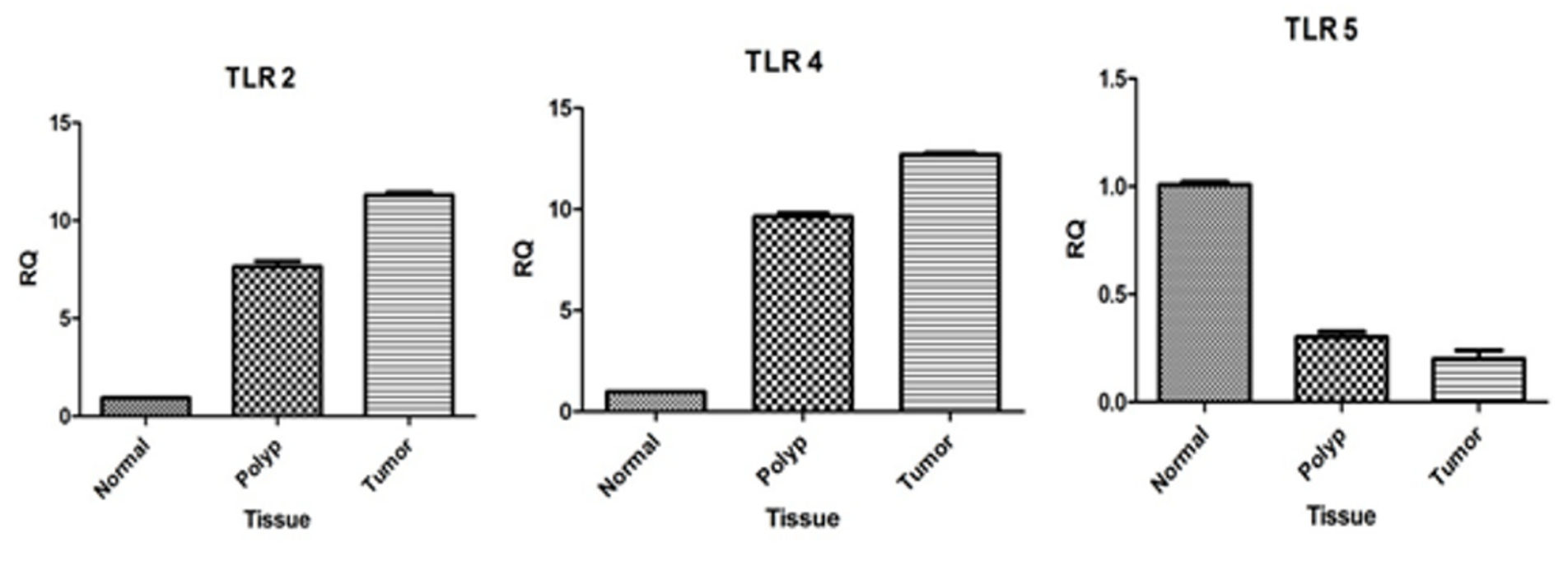

Figure 2

TLR2, TLR4 and TLR5 mRNA expression levels in AP and CRC cases compared to normal participants 